\section{FRATURA TRANSTROCANTERIANA}

Autoria: Sociedade Brasileira de Ortopedia e Traumatologia Colégio Brasileiro de Radiologia

Elaboração Final: 5 de novembro de 2007

Participantes: Roberto Sérgio de Tavares Canto, Marcos Sakaki, Itiro Susuki, Pedro Tucci, William Belangero, Maurício Kfuryi Jr. e Abdalla Y. Skaf

Canto RST, Sakaki M, Susuki I, Tucci P, Belangero W, Kfuri Jr $M$, Skaf AY

Descrição do método de coleta de evidência: Realizada pesquisa na base MEDLINE, pela interface MeSH (Medical Subject Heading). Os descritores utilizados foram: (old people AND trochanteric fracture AND conservative treatment AND outcomes) OR (old people AND trochanteric fracture AND (surgical treatment OR dynamic hip screw OR intramedullary nail OR Jewett plate OR Gotfried plate OR Ender Nail OR external fixator OR hip replacement) AND outcomes).

\section{Graus de recomendação e força de evidência:}

A: Estudos experimentais ou observacionais de melhor consistência.

B: Estudosexperimentaisou observacionaisdemenorconsistência.

C: Relatos de casos (estudos não controlados).

D: Opiniãodesprovidadeavaliaçãoc crítica, baseada em consensos, estudos fisiológicos ou modelos animais.

\section{Objetivo:}

Estabelecer orientação, com aplicabilidade para a realidade brasileira, em pontos controversos relacionados à fratura transtrocanteriana do quadril.

\section{Conflito de interesse:}

Nenhum conflito de interesse declarado.

\section{Introdução}

As fraturas transtrocanterianas são extracapsulares e ocorrem entre o grande e o pequeno trocânter. Perfazem um quarto das fraturas do quadril e incidem mais comumente no idoso mais velho. A sua incidência está aumentando de forma preocupante, juntamente com os custos socioeconômicos. O diagnóstico correto é realizado com uma radiografia no plano ântero-posterior, após tração gentil com rotação interna. 0 objetivo do tratamento é a estabilização precoce da fratura, com mínimo de morbidade adicional, para permitir o restabelecimento imediato da função. As comorbidades devem ser diagnosticadas e tratadas no préoperatório. As fraturas podem ser estáveis ou instáveis, pelo grau de cominuição da cortical póstero-medial que excede a fratura isolada do pequeno trocânter ou pelo traço invertido da fratura, que biomecanicamente se comporta como uma fratura subtrocantérica. A frequência das fraturas instáveis aumenta com a idade e a osteoporose. As fraturas estáveis apresentam uma taxa de complicação muito menor que as instáveis. A escolha do implante, uma boa redução e a exata colocação do mesmo com uma técnica cirúrgica esmerada permitem diminuir as complicações pós-operatórias das fraturas instáveis. O resultado cirúrgico de sucesso não significa necessariamente um resultado funcional equivalente, já que parte importante dos pacientes não recupera a condição deambulatória prévia à fratura.

Qual a utilidade da tração cutânea ou esquelética no préoperatório das fraturas transtrocantéricas?

O recurso da tração cutânea ou esquelética para alívio da dor no período pré-operatório não encontra suporte, uma vez que não há diferença quanto ao consumo de analgésico e na avaliação da dor, por escala analógica, com a utilização ou não da tração ${ }^{1}(\mathbf{A})$. As almofadas comuns ou especiais colocadas abaixo do quadril fraturado proporcionam o mesmo efeito analgésico que a tração cutânea ou esquelética ${ }^{1}(\mathbf{A})$. Quando comparados, os cuidados de enfermagem normais e sem tração com o uso de tração cutânea, não houve diferença quanto ao consumo de analgésicos, facilitação para o ato cirúrgico ou incidência de escaras²(A). 0 uso de tração cutânea ou esquelética no período pré-operatório das fraturas transtrocantéricas é contraindicado, relegando o seu uso a situações especiais ${ }^{3}(\mathbf{A})$.

0 tratamento cirúrgico precoce ( 24 horas) de pacientes com fratura transtrocanteriana do fêmur reduz a taxa de mortalidade?

A cirurgia realizada nas primeiras 24 horas não reduz a taxa de mortalidade durante o primeiro ano de vida dos pacientes com fratura transtrocanteriana ${ }^{4}(\mathbf{A}) .0$ paciente deve estar clinicamente compensado para ser submetido ao procedimento anestésico e cirúrgico ${ }^{5}(\mathbf{B})$. Os fatores relacionados ao aumento da mortalidade são: idade maior que 80 anos, presença de três ou mais comorbidades (principalmente cardíacas), rebaixamento mental, paciente institucionalizado e sexo masculino ${ }^{6}(\mathbf{A})$.

\section{Qual o melhor procedimento anestésico para o paciente com fratura transtrocanteriana do fêmur?}

Com relação ao tipo de anestesia, não há diferença quanto à mortalidade pós-operatória. Na anestesia espinal, é menor a tendência ao infarto do miocárdio, confusão mental, hipoxia e broncopneumonia ${ }^{\top}(\mathbf{A})$.

No pré-operatório, deve ser instalada precocemente infusão epidural contínua de anestésico local e opioide, para diminuir os eventos cardíacos adversos ${ }^{8}(\mathbf{A})$.

No intraoperatório, há evidência de maior número de episódios de hipotensão e queda do segmento ST, em anestesia geral e na espinal com dose única ${ }^{9}(\mathbf{A})$.

Não há diferença entre o bloqueio anestésico e a anestesia geral quanto à recuperação funcional e ao tempo de descarga de $\operatorname{peso}^{7}(\mathbf{A})$.

\section{Existem vantagens no uso da placa Medoff em relação ao dhs e às hastes cefalomedulares?}

A placa Medoff permite um deslizamento não só na região transtrocantérica como o DHS, mas também na região subtrocantérica, denominado dinamização biaxial. Os resultados de sua utilização quando comparados com o uso do DHS, sem e com placa de apoio trocantérico, e do DCS mostram igualdade em relação à incidência de falha da fixação e da capacidade 
de deambulação após um ano de seguimento ${ }^{10}(\mathbf{A})$. A dinamização biaxial proporciona maior encurtamento do fêmur no tratamento de fraturas transtrocanterianas instáveis, quando comparado com o DHS (15 mm contra $11 \mathrm{~mm}$ ), porém tal fato parece proteger a osteossíntese ao longo do processo de consolidação ${ }^{11}(\mathbf{A})$. Já no tratamento das fraturas subtrocantéricas, a utilização da placa de Medoff em modo de dinamização biaxial leva a elevado índice de falhas mecânicas, maior que o obtido com uso da haste Gamma ${ }^{12}(\mathbf{A})$.

\section{O lado da fratura transtrocantérica influencia nos resultados quando se utiliza o DHS?}

Ao se colocar o parafuso cefálico do DHS, o torque rotacional no sentido horário tende a desviar as fraturas transtrocantéricas de fêmures esquerdos, e reduzir as de fêmures direitos. A perda de redução pode ser observada pelo aparecimento na radiografia de uma saliência óssea anterior, que corresponde à porção distal e medial do colo desviada, com consequente diminuição da estabilidade. Medidas para evitar a rotação do colo devem ser tomadas na fixação de fraturas do fêmur esquerdo ${ }^{13}(\mathbf{B})$.

\section{Quais as vantagens e desvantagens em se utilizar a placa DHS com técnica minimamente invasiva?}

A osteossíntese com placa DHS minimamente invasiva no tratamento das fraturas transtrocantéricas leva a menor perda sanguínea, menor tempo cirúrgico e menos dor no pós-operatório, quando comparada à placa DHS utilizada de forma convencional, sem sacrifício da estabilidade da fratura e da consolidação ${ }^{14}(\mathbf{A})$.

\section{Atualmente, ainda existe lugar para o uso rotineiro da placa DHS no tratamento das fraturas trocantéricas?}

As fraturas transtrocantéricas podem ser divididas, segundo a classificação $A 0$, em estáveis (tipo $A 1$ ), instáveis com traço de fratura padrão (tipo A2) e instáveis com traço de obliquidade reversa (tipo A3). Nos tipos A1 e A2, a placa DHS, quando comparada às hastes cefalomedulares, proporciona resultados semeIhantes em relação a tempo cirúrgico, tempo de radioscopia, perda sanguínea, tempo de internação, mobilidade no pós-operatório, tempo de consolidação, perda de redução, mortalidade e resultado funcional ${ }^{15,16}(\mathbf{A})$. No entanto, a placa DHS não apresenta a fratura da diáfise do fêmur como complicação, que é relacionada ao pino $\operatorname{Gamma}^{17}(\mathbf{A})$. A placa $\mathrm{DHS}$ ainda tem indicação nas fraturas tipos $A 1$ e $A 2$. Já nas fraturas com traço reverso, tipo $A 3$, o uso de hastes cefalomedulares pode ser vantajoso ${ }^{18}(\mathbf{A})$.

\section{Nas fraturas trocantéricas estáveis, o DHS é o melhor método de fixação?}

Outros implantes, como a placa de Jewett e os pinos de Ender, mostram resultados semelhantes no tratamento das fraturas transtrocanterianas estáveis, com índice de falha em torno de $5 \%{ }^{19}(\mathbf{B})$.

\section{O uso do parafuso de compressão no sistema DHS melhora a estabilidade e favorece a consolidação das fraturas transtrocanterianas?}

O parafuso de compressão utilizado no sistema DHS, após a implantação do parafuso deslizante e da placa angulada, teria a finalidade de promover estabilização adicional da fixação, impactando os fragmentos proximal e distal, de uma fratura transtrocanteriana. Pacientes com fraturas transtrocanterianas submetidos a este tipo de osteossíntese, com e sem o uso do parafuso de compressão, não tiveram vantagens com o seu uso, em relação aos resultados do tratamento. Com o uso do parafuso de compressão ocorreu maior migração e consolidação em varo, em mulheres acima de 80 anos e com osteoporose. O uso deste parafuso não é recomendado para a fixação das fraturas transtrocantéricas ${ }^{20}(\mathbf{A})$.

O uso do parafuso de compressão no sistema DHS não está indicado após a colocação do implante ${ }^{20}(\mathbf{A})$. O seu uso ou não influi nos resultados de tratamento, além disso, o seu emprego em pacientes com osteoporose pode levar à migração do parafuso deslizante e à consolidação em varo.

\section{Em fraturas transtrocanterianas instáveis, é melhor a fixação com redução anatômica ou com osteotomia e medialização?}

Técnicas de fixação associadas a osteotomia valgizante e medialização foram descritas por Dimon Hughston (1976) e Sarmiento (1970) para melhorar a estabilidade em fraturas transtrocantéricas instáveis, à época, utilizando placas anguladas fixas (tipo Jewett). Posteriormente, com o advento dos sistemas dinâmicos (placa-parafuso tipo DHS), as fixações passaram a incorporar tais métodos. As duas possibilidades de fixação (com redução anatômica ou com osteotomia) têm resultados semelhantes quanto à consolidação e à recuperação funcional; as fixações com osteotomia demandam maior tempo cirúrgico e maior sangramento, sem interferência no resultado e quanto às complicações ${ }^{21}(\mathbf{A})$. Em outro estudo, foi observado menor tempo de hospitalização e melhores resultados quanto à recuperação funcional, quando foi obtida redução anatômica ${ }^{22}(\mathbf{B})$.

Por estas conclusões, não se observam vantagens na realização de osteotomias associadas a osteossíntese e seu uso não é recomendado para as fraturas transtrocantéricas instáveis.

\section{Qual o resultado esperado nas fraturas trocantéricas tratadas com a placa DHS?}

Após um ano de tratamento cirúrgico com DHS, tanto de fraturas estáveis como instáveis, 69\% dos pacientes estão vivos, sendo que $95 \%$ não referem dor ou apresentam dor leve, $85 \%$ voltam a mesma acomodação e 50\% retornam ao seu estado de mobilidade prévio à fratura. 0 índice de complicações relacionadas diretamente à fixação cirúrgica é de apenas 3,6\%, levando a reoperações em 2,6\% dos pacientes ${ }^{23}(\mathbf{B})$.

Existem situações em que as hastes podem ser empregadas com real vantagem sobre os dispositivos tradicionais (DHS, por exemplo)?

A análise dos estudos comparativos apresenta resultados favoráveis a ambos os sistemas, mas se deve notar que, nas fraturas estáveis, aparentemente não existe vantagem significante entre $\operatorname{ambos}^{24}(\mathbf{A})$; nas instáveis, os sistemas cefalomedulares são mais indicados; se houver necessidade de redução aberta, os resultados tendem mais para as fixações com pinos-placas 
deslizantes ${ }^{24}(\mathbf{A})$. O tempo cirúrgico e o sangramento mostramse semelhantes. Com relação às complicações, as hastes apresentam em maior número ${ }^{25}(\mathbf{A})$, sobretudo com fraturas diafisárias associadas $^{26}(\mathbf{A})$. Existem evidências ${ }^{24}(\mathbf{A})$ favorecendo o uso das hastes cefalomedulares nas fraturas de traço reverso ou cominutivas instáveis ${ }^{16}(\mathbf{A})$, tipo $\mathrm{A} 3$ ou fraturas transtrocantéricas com traço intertrocantérico. Nestes casos, as placas tipo DHS apresentam maior risco de complicações ${ }^{16}(\mathbf{A})$, entre elas perda de fixação, retardo de consolidação e quebras frequentes ${ }^{27}(\mathbf{A})$. Mesmo placas tipo DCS apresentam maior incidência complicações do que as hastes ${ }^{28}(\mathbf{A})$. 0 alto custo das hastes deve ser ponderado nas fraturas estáveis ou que possam ser fixadas com sistemas convencionais ${ }^{29}(\mathbf{A})$. Os resultados comparando hastes tipo Gamma nail e PFN são semelhantes ${ }^{30}(\mathbf{A})$.

\section{Qual o melhor método de fixação nas fraturas de traço reverso?}

O tratamento de fraturas instáveis de traço reverso do fêmur proximal deve ser conduzido da mesma forma que as fraturas subtrocantéricas ${ }^{31}(\mathbf{A})$. 0 uso de implantes do tipo placa-parafuso deslizante de quadril não oferece controle suficiente à tendência de medialização da diáfise. Recomenda-se, quando do uso destes sistemas para o tratamento deste tipo específico de fratura, a utilização complementar da placa de suporte trocantérico ${ }^{32}(\mathbf{A})$. 0 emprego de sistemas de placa de ângulo fixo de 95 graus constitui uma opção viável, seja como método de estabilidade relativa, adotando o princípio em ponte, no caso de fraturas cominutivas, ou o método de estabilidade absoluta, nos casos de traço simples e redução anatômica ${ }^{33}(\mathbf{B})$. Os implantes intramedulares constituem alternativa segura, viável de utilização percutânea, e oferecendo suficiente estabilidade à medialização da diáfise ${ }^{31}(\mathbf{A})$.

\section{Há necessidade do bloqueio distal da haste intramedular no tratamento das fraturas transtrocanterianas do fêmur?}

Pacientes submetidos a bloqueio distal de modo dinâmico, com dois parafusos na região da diáfise, quando comparados a pacientes submetidos a bloqueio de modo estático, após seguimento médio de 37 meses, toleraram melhor o bloqueio, e apresentaram menor número de casos com hipertrofia da cortical (1 de 34 versus 6 de 30) na ponta do implante. As demais queixas foram semelhantes nos dois modos de $\operatorname{bloqueio}^{34}(\mathbf{A})$.

\section{Quando está indicado o uso do fixador externo no tratamento de pacientes com fratura transtrocanteriana do fêmur?}

Com relação à indicação do fixador externo para o tratamento de fraturas transtrocanterianas, pode-se afirmar que: o fixador externo colocado sob anestesia local pode ser alternativa viável, segura e vantajosa para o tratamento de pacientes com alto risco cirúrgico (ASA 3 ou 4) $)^{35,36}$ (B). Fixador que utiliza pinos revestidos com hidroxiapatita pode ser considerado alternativa ao pino parafuso deslizante, com taxa de mortalidade, morbidade e tempo de consolidação semelhante, e, além disso, com menor custo $^{37}(\mathbf{B})$.

\section{Quando e em quais condições o tratamento conservador ainda poderá ser adotado?}

Nas condições atuais do tratamento cirúrgico, a abordagem conservadora, com repouso no leito associado à mobilização precoce, deve ser exclusivamente empregada diante da impossibilidade total de cirurgia, pois as taxas de mortalidade nos primeiros 30 dias podem ser 2,5 vezes maiores ${ }^{38}(\mathbf{B})$. 0 tratamento com tração contínua, mesmo esquelética, apresenta maus resultados quanto à mortalidade e à consolidação viciosa.

Somente em estudos antigos são citados resultados favoráveis ao tratamento conservador, sem mais suporte para seu emprego ${ }^{39}(\mathbf{B})$.

\section{Há benefícios em tratar fraturas transtrocanterianas com prótese total de quadril?}

Não há vantagem da artroplastia total do quadril comparada à fixação interna para o tratamento das fraturas transtrocanterianas. Não há diferença entre as duas formas de tratamento nas fraturas instáveis em termos de resultados, como tempo de internação hospitalar, tempo de descarga de peso ou complicações. Pacientes tratados com haste intramedular femoral proximal têm menor tempo operatório, menor perda sanguínea, menor taxa de mortalidade e menor custo hospitalar, quando comparados aos que recebem artroplastia ${ }^{40}(\mathbf{A})$. Pacientes muito idosos e com osteoporose avançada, com fraturas instáveis complexas da região proximal extra-articular do fêmur e que são elegíveis para a mobilização precoce podem se beneficiar do tratamento com $\operatorname{artroplastia}^{41}(\mathbf{A})$.

\section{Qual a morbi-mortalidade das fraturas transtrocanterianas?}

As fraturas transtrocanterianas estão relacionadas a índices elevados de morbidade e mortalidade. Após um ano da ocorrência da fratura, 20\% dos pacientes não se reintegraram à sua vida familiar e social prévia. Além disso, ao compararmos pacientes que sofreram e que não sofreram fratura, o primeiro grupo teve despesas com saúde três vezes maiores dentro do primeiro ano de tratamento. A mortalidade varia de $6 \%$ a $11 \%$ no primeiro mês, $14 \%$ a $36 \%$ no primeiro ano, sendo $15 \%$ maior do que a de indivíduos da mesma faixa etária que não sofreram a fratura ${ }^{42}(\mathbf{A})$.

\section{Referências}

1. Resch S, Bjärnetoft B, Thorngren KG. Preoperative skin traction or pillow nursing in hip fractures: a prospective, randomized study in 123 patients. Disabil Rehabil 2005;27:1191-5.

2. Anderson GH, Harper WM, Connolly CD, Badham J, Goodrich N, Gregg PJ. Preoperative skin traction for fractures of the proximal femur. A randomised prospective trial. J Bone Joint Surg Br 1993;75:794-6.

3. Beaupre LA, Jones CA, Saunders LD, Johnston DW, Buckingham J, Majumdar

SR. Best practices for elderly hip fracture patients. A systematic overview of the evidence. J Gen Intern Med 2005;20:1019-25.

4. Vidán M, Serra JA, Moreno C, Riquelme G, Ortiz J. Efficacy of a comprehensive geriatric intervention in older patients hospitalized for hip fracture: a randomized, controlled trial. J Am Geriatr Soc 2005;53:1476-82.

5. Orosz GM, Magaziner J, Hannan EL, Morrison RS, Koval K, Gilbert M, et al. Association of timing of surgery for hip fracture and patient outcomes. JAMA 2004;291:1738-43.

6. Roche JJ, Wenn RT, Sahota O, Moran CG. Effect of comorbidities and postoperative complications on mortality after hip fracture in elderly people: prospective observational cohort study. BMJ 2005;331:374.

7. Valentin N, Lomholt B, Jensen JS, Hejgaard N, Kreiner S. Spinal or general anaesthesia for surgery of the fractured hip? A prospective study of mortality in 578 patients. Br J Anaesth 1986;58:284-91. 
8. Matot I, Oppenheim-Eden A, Ratrot R, Baranova J, Davidson E, Eylon S, et al. Preoperative cardiac events in elderly patients with hip fracture randomized to epidural or conventional analgesia. Anesthesiology 2003;98:156-63.

9. Beaupre LA, Jones CA, Saunders LD, Johnston DW, Buckingham J, Majumdar SR. Best practices for elderly hip fracture patients. A systematic overview of the evidence. J Gen Internal Med 2005;20:1019-25.

10. Lunsjö K, Ceder L, Thorngren KG, Skytting B, Tidermark J, Berntson PO, et al. Extramedullary fixation of 569 unstable intertrochanteric fractures: a randomized multicenter trial of the Medoff sliding plate versus three other screw-plate systems. Acta Orthop Scand 2001;72:133-40.

11. Olsson O, Ceder L, Hauggaard A. Femoral shortening in intertrochanteric fractures. A comparison between the Medoff sliding plate and the compression hip screw. J Bone Joint Surg Br 2001;83:572-8.

12. Miedel R, Ponzer S, Tornkvist H, Söderqvist A, Tidermark J. The standard Gamma nail or the Medoff sliding plate for unstable trochanteric and subtrochanteric fractures. A randomized, controlled trial. J Bone Joint Surg Br 2005;87:68-75.

Gamma nail and the Ambi hip screw. Aust N Z J Surg 1996;66:151-5.

26. Herrera A, Domingo LJ, Calvo A, Martínez A, Cuenca J. A comparative study of trochanteric fractures treated with the Gamma nail or the proximal femoral nail. Int Orthop 2002;26:365-9.

27. Giraud B, Dehoux E, Jovenin N, Madi K, Harisboure A, Usandizaga G, et al. Pertrochanteric fractures: a randomized prospective study comparing dynamic screw plate and intramedullary fixation. Rev Chir Orthop Reparatrice Appar Mot 2005;91:732-6.

28. Sadowski C, Lübbeke A, Saudan M, Riand N, Stern R, Hoffmeyer P. Treatment of reverse oblique and transverse intertrochanteric fractures with use of na intramedullary nail or a 95 degrees screwplate: a prospective, randomized study. J

Bone Joint Surg Am 2002;84:372-81.

29. Saudan M, Lübbeke A, Sadowski C, Riand N, Stern R, Hoffmeyer P. Pertrochanteric fractures: is there an advantage to na intramedullary nail?: a randomized, prospective study of 206 patients comparing the dynamic hip screw and proximal femoral nail. J Orthop Trauma 2002;16:386-93.

30. Schipper IB, Steyerberg EW, Castelein RM, van der Heijden FH, den Hoed PT,

Kerver AJ, et al. Treatment of unstable trochanteric fractures. Randomised comparison of the gamma nail and the proximal femoral nail. J Bone Joint Surg $\mathrm{Br}$ 2004;86:86-94.

31. Kregor PJ, Obremskey WT, Kreder HJ, Swiontkowski MF; Evidence-Based Orthopaedic Trauma Working Group. Unstable pertrochanteric femoral fractures. J Orthop Trauma 2005;19:63-6.
32. Parker MJ, Handoll HH. Gamma and other cephalocondylic intramedullary nails versus extramedullary implants for extracapsular hip fractures in adults. Cochrane Database Syst Rev 2005;(4):CD000093.

33. Brammar TJ, Kendrew J, Khan RJ, Parker MJ. Reverse obliquity and transverse fractures of the trochanteric region of the femur; a review of 101 cases. Injury 2005;36:851-7.

34. Hardy DC, Drossos K. Slotted intramedullary hip screw nails reduce proximal

mechanical unloading. Clin Orthop Relat Res 2003;406:176-84.

35. Ozdemir H, Dabak TK, Urgüden M, Gür S. A different treatment modality for trochanteric fractures of the femur in surgical high-risk patients: a clinical study of 44 patients with 21-month follow-up. Arch Orthop Trauma Surg 2003;123: 538-43.

36. Devgan A, Sangwan SS. External fixator in the management of trochanteric fractures in high risk geriatric patients: a friend to the elderly. Indian J Med Sci 2002;56:385-90.

37. Moroni A, Faldini C, Pegreffi F, Hoang-Kim A, Vannini F, Giannini S. Dynamic hip screw compared with external fixation for treatment of osteoporotic pertrochanteric fractures. A prospective, randomized study. J Bone Joint Surg Am 2005;87:753-9.

38. Jain R, Basinski A, Kreder HJ. Nonoperative treatment of hip fractures. Int Orthop 2003;27:11-7.

39. Bong SC, Lau HK, Leong JC, Fang D, Lau MT. The treatment of unstable intertrochanteric fractures of the hip: a prospective trial of 150 cases. Injury 1981;13:139-46.

40. KimSY, Kim YG, Hwang JK. Cementlesscalcar-replacementhemiarthroplasty compared with intramedullary fixation of unstable intertrochanteric fractures. A prospective, randomized study. J Bone Joint Surg Am 2005;87:2186-92. 41. Stappaerts KH, Deldycke J, Broos PL, Staes FF, Rommens PM, Claes P. Treatment of unstable peritrochanteric fractures in elderly patients with a compression hip screw or with the Vandeputte (VDP) endoprosthesis: a prospective randomized study. J Orthop Trauma 1995;9:292-7.

42. Hagsten B, Svensson O, Gardulf A. Health-related quality of life and selfreported ability concerning $A D L$ and IADL after hip fracture: a randomized trial. Acta Orthop 2006;77:114-9.

O texto completo da Diretriz: Fratura Transtrocanteriana está disponível nos sites: www. projetodiretrizes.org.br e www.amb.org.br. 